\title{
Power-free Values of Strongly $Q$-additive Functions
}

\author{
Karam Aloui*, Mohamed Mkaouar and Walid Wannes
}

\begin{abstract}
Let $f$ be a strongly $q$-additive function with integer values. Given an integer $k \geq 2$, we try to estimate the number of positive integers $n \leq N$ (resp. primes $p \leq N$ ) for which $f(n)$ is $k$-free (resp. $f(p)$ is $k$-free).
\end{abstract}

\section{Introduction}

Throughout this paper, $\mathbb{P}, \mathbb{N}, \mathbb{N}_{0}, \mathbb{Z}$ and $\mathbb{R}$ denote respectively the sets of prime numbers, positive integers, nonnegative integers, integers and real numbers. $p$ denotes always a prime number, $\zeta$ is the Riemann zeta function and $\varphi$ is the Euler totient function. For any real number $x$, we define $\lfloor x\rfloor$ as the largest integer smaller than or equal to $x,\|x\|$ is the distance from $x$ to the nearest integer, $e(x)=\exp (2 \pi i x), \pi(x)$ is the number of primes $p \leq x$ and $\pi(x ; m, l)$ denotes the number of primes $p \leq x$ that are congruent to $l \bmod m$ for some coprime integers $l, m(m \in \mathbb{N})$. The notations $(a, b)$ and $[a, b]$ refer respectively to the greatest common divisor and the least common multiple of the integers $a$ and $b$ and more generally, given $n$ integers $a_{1}, \ldots, a_{n}$, we denote by $\left(a_{1}, \ldots, a_{n}\right)$ their greatest common divisor. We denote by $|\mathcal{A}|$ the number of elements of a given set $\mathcal{A}$. We recall the Vinogradov's notation $U \ll V$ equivalent to the Bachmann-Landau notation $U=O(V)$ for complex valued functions $U$ and $V$ where the implied constants in the symbols " $O$ ", "«" are absolute. If the implied constants depend on certain parameters $\alpha, \beta, \ldots$ (but on no other parameters), then we write $U(N)=O_{\alpha, \beta, \ldots}(V(N))$ and $U(N) \ll_{\alpha, \beta, \ldots} V(N)$.

Let $k \geq 2$ be an integer, a positive integer $n$ is said to be $k$-free if for each prime $p$, one has $p^{k} \nmid n$. Let $\mu_{k}$ denote the characteristic function of $k$-free integers, then one has the identity:

$$
\mu_{k}(n)=\sum_{d^{k} \mid n} \mu(d)
$$

Received June 10, 2018; Accepted December 16, 2018.

Communicated by Yifan Yang.

2010 Mathematics Subject Classification. 11A63, 11L03, 11N05, 11N69.

Key words and phrases. $k$-free numbers, strongly $q$-additive function, exponential sums, prime numbers. Aloui was supported by the binational research project MuDeRa (ANR-14-CE34-0009) which is funded by the French and Austrian Science Funds ANR and FWF.

Wannes was supported by the FWF Grant F5510 within the special research area (SFB) quasi Monte Carlo Methods: Theory and Applications.

*Corresponding author. 
where $\mu$ is the Möbius function.

Let $M_{k}(x)=\sum_{n \leq x} \mu_{k}(n)$ be the number of $k$-free integers $\leq x$, using elementary arguments, one can assert

$$
M_{k}(x)=\frac{x}{\zeta(k)}+O\left(x^{1 / k}\right)
$$

Several authors worked intensively to improve the error term and the best unconditional result remains that due to Walfisz [28] who showed that, uniformly on $k$, there exists an absolute constant $c$ such that

$$
M_{k}(x)=\frac{x}{\zeta(k)}+O\left(x^{1 / k} \exp \left(-c k^{-8 / 5} \frac{(\log x)^{3 / 5}}{(\log \log x)^{1 / 5}}\right)\right) .
$$

Under the Riemann hypothesis, Montgomery and Vaughan 22 proved that for every $\varepsilon>0$

$$
M_{k}(x)-\frac{x}{\zeta(k)}=O\left(x^{1 /(k+1)+\varepsilon}\right) .
$$

This error term was improved by many authors, still under the Riemann hypothesis, such as Graham, Baker and Pintz, Jia, Graham and Pintz and Baker and Powell [1, 2, 9, 10, 13 , 14]. Their error terms are of the form $x^{f(k)}$ for some $f(k) \sim 1 / k$ as $k \rightarrow+\infty$. The best bound for square-free numbers $(k=2)$ is that of Jia [14] of the form $x^{17 / 54+\varepsilon}$.

Recently, Meng [19] studied the analytic properties of the $\zeta$ function in order to prove that, under the Riemann hypothesis, we have

$$
M_{k}(x)-\frac{x}{\zeta(k)} \ll_{k, \varepsilon} x^{1 /(2 k)}(\log x)^{1 / 2-1 /(2 k)+\varepsilon}
$$

except on a set of finite logarithmic measure. Actually, 25 provides a nice survey about this topic for interested readers.

Another question related to $k$-freeness is the asymptotic behaviour of

$$
\mid\{n \leq x, f(n) \text { is } k \text {-free }\} \mid
$$

for any function $f: \mathbb{N} \rightarrow \mathbb{Z}$. We mention a result of Mirsky [20] that deals with the case $f(t)=\mathbb{1}_{\mathbb{P}}(t)+c$ where $\mathbb{1}_{\mathbb{P}}$ denotes the characteristic function of the primes and $c \in \mathbb{Z}$. In this case, we get for any real number $B>1$,

$$
\mid\{p \leq x, p+c \text { is } k \text {-free }\} \mid=\beta_{\mathrm{Ar}} \pi(x)+O\left(\frac{x}{\log ^{B} x}\right),
$$

where

$$
\beta_{\mathrm{Ar}}=\prod_{p \in \mathbb{P}}\left(1-\frac{1}{p^{k}(p-1)}\right)
$$


is called Artin's $k$-dimensional constant. In the same context, Heath-Brown 12$]$ considered an irreducible polynomial of the form $f(X)=X^{d}+c \in \mathbb{Z}[X]$ and showed that for $k \geq$ $(5 / d+3) / 9$, there exists a constant $\delta(d)$ such that

$$
\mid\{n \leq x, f(n) \text { is } k \text {-free }\} \mid=C(f, k) x+O\left(x^{1-\delta(d)}\right) .
$$

Here, the constant $C(f, k)$ is given by

$$
C(f, k)=\prod_{p}\left(1-\rho_{f}\left(p^{k}\right) p^{-k}\right)
$$

where

$$
\rho_{f}(d)=|\{n \bmod d: d \mid f(n)\}| .
$$

Using Heath-Brown's method, Reuss [24] provided an asymptotic formula for the number of primes $p \leq x$ such that $f(p)$ is $(d-1)$-free, where $f$ is an irreducible polynomial of degree $d \geq 3$ with no fixed prime divisors.

Now, let $q \geq 2$ be an integer. Then we can represent every positive integer $n$ in a unique way as

$$
n=\sum_{0 \leq j \leq \nu} n_{j} q^{j}, \quad n_{j} \in\{0, \ldots, q-1\} \text { and } n_{\nu} \neq 0 .
$$

This representation is called the $q$-ary expansion of $n$ with respect to base $q$; and the set $\{0, \ldots, q-1\}$ is called the set of digits.

According to (1.4), we may define the sum of digits of the integer $n$ by

$$
S_{q}(n)=\sum_{0 \leq j \leq \nu} n_{j}
$$

which might also be denoted by $S(n)$ if there is no risk of confusion.

In 1967, Gelfond [8] wrote a survey on strongly $q$-additive functions, namely the functions $f: \mathbb{N} \rightarrow \mathbb{R}$ that satisfy $f\left(a q^{i}+b\right)=f(a)+f(b)$, for all $(a, i, b) \in \mathbb{N}^{2} \times \mathbb{N}_{0}$ and $0 \leq b<q^{i}$. In particular, we have $f(0)=0$ and

$$
f(n)=\sum_{0 \leq j \leq \nu} f\left(n_{j}\right)
$$

meaning that a strongly $q$-additive function is completely determined by its values on the integers $1, \ldots, q-1$. A simple example of such a function is the sum of digits function. Besides, Gelfond's paper dealt with a non trivial estimate of the exponential sum

$$
\sum_{n \leq x} e\left(\frac{l}{m} S_{q}(n)+\beta n\right)
$$

where $\beta \in \mathbb{R}, q, l$ and $m$ are integers $\geq 2$ such that $1 \leq l<m$ and $(q-1, m)=1$. He proved also the following theorem concerning the sum of digits function along $k$-free integers: 
Theorem 1.1. (see [8, Théorème II]) Let $q, m, k \geq 2$ and $l$ be integers such that ( $q-$ $1, m)=1$, then we have

$$
\mid\left\{n \leq x, n \text { is } k \text {-free, } S_{q}(n) \equiv l \bmod m\right\} \mid=\frac{x}{m \zeta(k)}+O_{q}\left(N^{\lambda_{1}}\right),
$$

where $\lambda_{1}=\frac{1+(k-1) \lambda_{0}}{k}$ and $\lambda_{0}=\frac{1}{2 \log q} \log \frac{q \sin (\pi /(2 m))}{\sin (\pi /(2 m q))}$.

The strongly $q$-additive functions have been extensively discussed in the literature, mainly their asymptotic distribution (see for instance $4,5,16,18,21]$ ). For further information and details, the reader is advised to refer to the interesting survey of Pappalardi [23].

Our goal in this paper is to consider the analogue version of problem (1.1) in the case of strongly $q$-additive functions. Indeed, we try to estimate the asymptotic behaviour of

$$
\mid\{n \leq x, f(n) \text { is } k \text {-free }\} \mid
$$

and

$$
\mid\{p \leq x, f(p) \text { is } k \text {-free }\} \mid, \quad \text { whenever } q=2
$$

where $f$ is a strongly $q$-additive function with integer values.

In order to achieve such estimates, we should introduce the class of digital functions, i.e., functions $f: \mathbb{N} \rightarrow \mathbb{R}$ such that there exists $a_{0}, \ldots, a_{q-1} \in \mathbb{R}$ satisfying

$$
f(n)=\sum_{0 \leq k<q} a_{k}|n|_{k} \quad \text { for every } n \in \mathbb{N}
$$

where we denote, according to 1.4 ,

$$
|n|_{k}=\left|\left\{0 \leq j \leq \nu \mid n_{j}=k\right\}\right| .
$$

The sum of digits function is a trivial example of a digital function since

$$
S_{q}(n)=\sum_{0 \leq k<q} k|n|_{k}
$$

Note that every strongly $q$-additive function is digital since, according to (1.5),

$$
f(n)=\sum_{0 \leq j \leq \nu} f\left(n_{j}\right)=\sum_{1 \leq k<q} f(k)|n|_{k} .
$$

In particular, $a_{k}=f(k), \forall k \in\{0, \ldots, q-1\}$.

Conversely, every digital function $f(n)=\sum_{1 \leq k<q} f(k)|n|_{k}$ is strongly $q$-additive.

We shall denote by $|\cdot|_{k}$ the function that assigns to each positive integer $n$, the integer $|n|_{k}$ already defined in (1.6), $\mathcal{F}$ is the set of digital functions $f=\sum_{0 \leq k<q} a_{k}|\cdot|_{k}$ and $\mathcal{F}_{0}$ is the subset of $\mathcal{F}$ such that the sequence $a_{0}, \ldots, a_{q-1}$ is an arithmetic progression modulo 
1 (i.e., there exists some $r \in \mathbb{R}$ such that $a_{j} \equiv a_{0}+j r \bmod 1$ ). It is easily seen that $S_{q}(\cdot) \in \mathcal{F}_{0}$.

Let $f=\sum_{0 \leq k<q} a_{k}|\cdot|_{k} \in \mathcal{F}$, we define the real number $\lambda_{q}(f)$ as follows:

$$
\lambda_{q}(f)= \begin{cases}c_{0} \min _{t \in \mathbb{R}} \sum_{0 \leq j<i<q}\left\|a_{i}-a_{j}-(i-j) t\right\|^{2} & \text { if } f \notin \mathcal{F}_{0}, \\ c_{0}\left\|(q-1)\left(a_{1}-a_{0}\right)\right\|^{2} & \text { if } f \in \mathcal{F}_{0},\end{cases}
$$

where $c_{0}$ is a constant depending only on $q$ (defined in $[17$, Théorème A]). Actually, the authors showed that $\lambda_{q}(f)>0$. Furthermore, they extended the theorems of Hadamardde La Vallée Poussin (the Prime Number Theorem) and Vinogradov (see [6, 11, 27]) to the case of prime numbers under digital constraints. Their method relies on the estimate of exponential sums such as the following

Theorem 1.2. (see $[17$, Théorème A]) Let $q \geq 2$ be an integer and $f \in \mathcal{F}$. Then, for all $x \geq 2$ and $\beta \in \mathbb{R}$, we have

$$
\sum_{n \leq x} \Lambda(n) e(f(n)+\beta n) \ll_{q} x^{1-\lambda_{q}(f)}(\log x)^{4},
$$

where $\lambda_{q}(f)$ is the constant defined in (1.7).

We can find a generalized version of Theorem 1.2 in [21].

Our work is organized as follows: In Section 2, we give some preliminary results including the estimate of the exponential sum $\sum_{1 \leq n \leq x} e(\alpha n+\beta f(n))$, where $f$ is a digital function. Moreover, we provide a theorem investigating the asymptotic behaviour of

$$
\Xi=|\{1 \leq n \leq x: n \equiv l \bmod r, f(n) \equiv a \bmod b\}| .
$$

Indeed, we affirm that there exists a set $J_{1}$ and some positive constant $\theta_{f, b, q}$ such that

$$
\Xi=\frac{x}{r b} \sum_{(j, h) \in J_{1}} \cos \left(2 \pi\left(\frac{l}{r} j+\frac{a}{b} h\right)\right)+O_{q}\left(x^{1-\theta_{f, b, q}}\right),
$$

generalizing results known from Gelfond's work 8].

Section 3 deals with a problem of $k$-freeness on a subclass of strongly $q$-additive functions. Indeed, let $\mathcal{F}_{q}^{1}$ be the set of strongly $q$-additive functions with integer values such that $f(j)=1$ for some $j \in\{1, \ldots, q-1\}$ and let $\mathcal{F}_{q}^{+}$be the set of strongly $q$-additive functions $f$ such that

$$
f=\sum_{1 \leq k<q} a_{k}|\cdot|_{k}, \quad a_{1}, \ldots, a_{q-1} \in \mathbb{Z} \text { and }\left(a_{1}, \ldots, a_{q-1}\right)=1 .
$$

These sets will be of major interest in our article since they include the sum of digits function (for instance) and they enable estimates involving congruences.

Basically, we find the following theorem. 
Theorem 1.3. Let $k \geq 2$ be an integer and let $f \in \mathcal{F}_{q}^{1}$. Then, we have

$$
\mid\{1 \leq n \leq x, f(n) \text { is } k \text {-free }\} \mid=\frac{x}{\zeta(k)}+O\left(\frac{x}{\log ^{k-1} x}\right) .
$$

The second theorem states the corresponding estimate along prime numbers for completely 2-additive functions. Let $f \in \mathcal{F}_{q}^{+}$and let $b \geq 2$ be an integer, we set the integer $d=d_{f, b, q} \geq 1$ as the greatest divisor of $(b, q-1)$ such that $(f(1), d)=1$ and for all nonnegative integers $n$

$$
f(n) \equiv f(1) S_{q}(n) \equiv f(1) n \bmod d .
$$

Obviously, such an integer must exist (1 is the least positive integer satisfying these assumptions).

By using the result of Martin-Mauduit-Rivat (see [17, Lemme 6]), we have for all $j \in J_{2}=\{0 \leq j<b, b / d \nmid j\}$,

$$
\sum_{p \leq N} e\left(\frac{j}{b} f(p)+r p\right) \ll_{q} N^{1-\sigma_{f, b, q}}(\log N)^{3} .
$$

Using elementary means and the above result, Martin, Mauduit and Rivat [17] proved the following

Theorem 1.4. (see 17, Théorème 3]) Let $q, b \geq 2$ be integers, let $f \in \mathcal{F}_{q}^{+}$and $d=d_{f, b, q}$ be the integer defined in (1.9). Let $c=f(1)^{*}$ be the inverse of $f(1)$ modulo $d$. Then, for every $a \in \mathbb{Z}$, there exists a positive constant $\sigma_{f, b, q}$ such that

$$
|\{p \leq x, f(p) \equiv a \bmod b\}|= \begin{cases}0 \text { or } 1 & \text { if }(a, d)>1 \\ \frac{d}{b} \pi(x ; d, a c)+O_{q}\left((\log x)^{3} x^{\left.1-\sigma_{f, b, q}\right)}\right. & \text { otherwise. }\end{cases}
$$

In view of the above, our second theorem is the following

Theorem 1.5. Let $k \geq 2$ be an integer, let $f \in \mathcal{F}_{2}^{+}$. Then,

$$
\mid\{p \leq x, f(p) \text { is } k \text {-free }\} \mid=\frac{\pi(x)}{\zeta(k)}+O\left(\frac{x}{\log ^{k} x}\right) .
$$

Section 4 is devoted to the distribution of $k$-free numbers in congruence classes with respect to strongly $q$-additive functions. Indeed, we look for

$$
D_{b}(x)=\mid\{n \leq x, n \text { is } k \text {-free, } f(n) \equiv a \bmod b\} \mid,
$$

which extends Theorem 1.1 and in the same vein

$$
\mid\{p \leq x, p+c \text { is } k \text {-free, } f(p) \equiv a \bmod b\} \mid
$$


is extending 1.2 . In fact, we prove that

$$
D_{b}(x)=\frac{x}{b \zeta(k)} \sum_{h \in \mathcal{J}} \cos \left(2 \pi \frac{a}{b} h\right)+O_{q}\left(x^{\widetilde{\theta}_{f, b, q}}\right),
$$

where

$$
\mathcal{J}=\left\{0 \leq h<b, \frac{h}{b} f(\ell) \in \mathbb{Z}, \forall \ell \in\{1, \ldots, q-1\}\right\}
$$

Martin-Mauduit-Rivat provided an extended version of Theorem 1.4 in the following form.

Theorem 1.6. (see 17, Théorème 5]) Let the setup be as in Theorem 1.4. For all integers $k \geq 2$ and $l$,a, we have

$$
\sum_{\substack{p \leq x \\ p \equiv l=0 \\ f(p) \equiv a \bmod b}} 1= \begin{cases}0 & \text { if } l \not \equiv b c \bmod (k, d), \\ \frac{d}{b} \pi(x ;[k, d], v)+O\left((\log x)^{3} x^{\left.1-\sigma_{f, b, q}\right)}\right. & \text { otherwise }\end{cases}
$$

where $v$ is a solution of the congruence system

$$
v \equiv l \bmod k, \quad v \equiv b c \bmod d
$$

and $\sigma_{f, b, q}$ is the constant stated in Theorem 1.4 .

In particular, if $d=1$, we have

$$
|\{p \leq x, p \equiv l \bmod k, f(p) \equiv a \bmod b\}|=\frac{\pi(x ; k, l)}{b}+O\left((\log x)^{3} x^{1-\sigma_{f, b, q}}\right) .
$$

Relying on Theorem 1.6, we shall find the following estimate

$$
\mid\{p \leq x, p+c \text { is } k \text {-free, } f(p) \equiv a \bmod b\} \mid=\frac{\beta_{\mathrm{Ar}}}{b} \pi(x)+O\left(\frac{x}{\log ^{2} x}\right),
$$

where $\beta_{\mathrm{Ar}}$ is the Artin's $k$-dimensional constant defined in 1.3 .

Finally, in the last section, we combine the results of Sections 3 and 4 with a lemma of Banks, Harman and Shparlinski [3], in order to evaluate the cardinality of the set

$$
\{n \leq x, f(\mathcal{P}(n)) \text { is } k \text {-free }\}
$$

where $\mathcal{P}(n)$ is the largest prime factor of $n$ and $f$ is a strongly 2-additive function. As a matter of fact, we prove that in this case, we have

$$
\mid\{n \leq x, f(\mathcal{P}(n)) \text { is } k \text {-free }\} \mid=\frac{x}{\zeta(k)}+O\left(x \log x \exp \left(-C \log ^{1 / 3} x\right)\right)
$$

for some positive constant $C$. 
2. An estimate of exponential sums involving digital functions

Given a digital function $f=\sum_{0 \leq k<q} a_{k}|\cdot|_{k}$, we set $\phi$ the periodic function of period 1 defined by

$$
\phi(t)=\left|\sum_{0 \leq k<q} e\left(a_{k}-k t\right)\right|, \quad t \in \mathbb{R} .
$$

Given $\lambda \in \mathbb{N}$, we introduce the function $|\cdot|_{\lambda, k}$ defined by

$$
|n|_{\lambda, k}=\left|\left\{0 \leq j<\lambda, n_{j}=k\right\}\right| \text { with respect to } 1.4 \text {, }
$$

and the function $f_{\lambda}$ defined by

$$
f_{\lambda}(n)=\sum_{0 \leq k<q} a_{k}|n|_{\lambda, k}
$$

Set the function $\Phi_{\lambda}(t)=\sum_{0 \leq l<q^{\lambda}} e\left(f_{\lambda}(l)+l t\right), t \in \mathbb{R}$.

We introduce a classic lemma, estimating some exponential sums that will be needed later and whose proof can be found in [16, Lemme 12].

Lemma 2.1. Let $f \in \mathcal{F}, \lambda \in \mathbb{N}$. For $t \in \mathbb{R}, N \geq 1$, we have

$$
\left|\sum_{0<n<N} e(f(n)+n t)\right| \leq 2(q-1) \sum_{\lambda \leq \frac{\log N}{\log q}}\left|\Phi_{\lambda}(t)\right| .
$$

Now, we set the real number $\gamma_{q}(f)$ defined by

$$
q^{\gamma_{q}(f)}=\max _{t \in \mathbb{R}} \sqrt{\phi(t) \phi(q t)}
$$

Martin, Mauduit and Rivat could find significant bounds for $\gamma_{q}(f)$ in 16 , Lemmes 8, 10, 11]. These bounds shall be useful in order to state the following result.

Theorem 2.2. Let $\alpha$ be a real number, let $f \in \mathcal{F}_{0}$ such that $f(0)=0$ and $(q-1) f(1) \in \mathbb{Z}$. Then,

$$
\sum_{1 \leq n \leq x} e(\alpha n+f(n))= \begin{cases}\lfloor x\rfloor & \text { if }(\alpha+f(1)) \in \mathbb{Z}, \\ e(\alpha+f(1)) \frac{\sin (\pi\lfloor x\rfloor(\alpha+f(1)))}{\sin (\pi(\alpha+f(1)))} & \text { otherwise. }\end{cases}
$$

Otherwise, we have

$$
\sum_{1 \leq n \leq x} e(\alpha n+f(n)) \ll_{q} x^{\gamma_{q}(f)}
$$


where

$$
\gamma_{q}(f) \leq \begin{cases}1-\frac{16}{q^{2}(q-1) \log q} \sigma_{q}(f) & \text { if } f \notin \mathcal{F}_{0}, \\ 1-\frac{4\|(q-1) f(1)\|^{2}}{q(q+\sqrt{2}-1)^{2} \log q} & \text { if } f \in \mathcal{F}_{0} \text { and }(q-1) f(1) \in \mathbb{R} \backslash \mathbb{Z}\end{cases}
$$

and $\sigma_{q}(f)=\min _{t \in \mathbb{R}} \sum_{0 \leq j<i<q}\|(f(i)-f(j))-(i-j) t\|^{2}>0$ according to the identity (8) of $[16$.

Proof. We set $N=\lfloor x\rfloor=\sum_{h=0}^{\nu} a_{h} q^{h}$ with $a_{\nu} \neq 0$ (then in particular $\nu=\left\lfloor\frac{\log x}{\log q}\right\rfloor$ ). We shall deal with two cases depending on whether $f \in \mathcal{F}_{0}$ or not.

Case 1. If $f \in \mathcal{F}_{0}$, the sequence $0, f(1), \ldots, f(q-1)$ is an arithmetic progression modulo 1 . Thus, we argue according to $(q-1) f(1) \in \mathbb{Z}$ or not.

So, if $(q-1) f(1)=l \in \mathbb{Z}$ then we write $f(j) \equiv j \frac{l}{q-1} \bmod 1$, for $0 \leq j<q$, and reminding that $S_{q}(n) \equiv n \bmod (q-1)$, for every positive integer $n$, we get

$$
\begin{aligned}
\sum_{1 \leq n \leq x} e(\alpha n+f(n)) & =\sum_{1 \leq n \leq x} e\left(\alpha n+f(1)|n|_{1}+\cdots+f(q-1)|n|_{q-1}\right) \\
& =\sum_{1 \leq n \leq x} e\left(\alpha n+\frac{l}{q-1}|n|_{1}+\frac{2 l}{q-1}|n|_{2}+\cdots+l|n|_{q-1}\right) \\
& =\sum_{1 \leq n \leq x} e\left(\alpha n+\frac{l}{q-1} S(n)\right) \\
& =\sum_{1 \leq n \leq x} e\left(\left(\alpha+\frac{l}{q-1}\right) n\right) \\
& = \begin{cases}\lfloor x\rfloor & \text { if }(\alpha+l /(q-1)) \in \mathbb{Z}, \\
e(\alpha+l /(q-1)) \frac{\sin \left(\pi\lfloor x\rfloor\left(\alpha+\frac{l}{q-1}\right)\right)}{\sin \left(\pi\left(\alpha+\frac{l}{q-1}\right)\right)} & \text { otherwise. }\end{cases}
\end{aligned}
$$

Furthermore, if $(q-1) f(1) \notin \mathbb{Z}$, then according to Lemma 2.1, we have

$$
\left|\sum_{1 \leq n \leq x} e(\alpha n+f(n))\right| \leq 1+2(q-1) \sum_{\lambda \leq \frac{\log x}{\log q}}\left|\Phi_{\lambda}(\alpha)\right|,
$$

where

$$
\Phi_{\lambda}(\alpha)=\sum_{0 \leq l<q^{\lambda}} e\left(\alpha l+\sum_{0 \leq k<q} f(k)|l|_{\lambda, k}\right) .
$$

But, it is easy to see that

$$
\left|\Phi_{\lambda}(\alpha)\right|=\prod_{0 \leq j<\lambda} \phi\left(-q^{j} \alpha\right)=\prod_{0 \leq j<\lambda}\left|\sum_{0 \leq k<q} e\left(f(k)+\alpha k q^{j}\right)\right| .
$$


We refer back to 2.1 so to get

$$
\begin{aligned}
\prod_{0 \leq j<\lambda} \phi\left(-q^{j} \alpha\right) & \leq q \prod_{0 \leq j \leq\lfloor(\lambda-1) / 2\rfloor} \phi\left(-q^{2 j} \alpha\right) \phi\left(-q^{2 j+1} \alpha\right) \\
& \leq q^{2 \gamma_{q}(f)\lfloor(\lambda+1) / 2\rfloor+1} \leq q^{\gamma_{q}(f)(\lambda+1)+1} .
\end{aligned}
$$

Putting this in 2.3 , we find

$$
\left|\sum_{1 \leq n \leq x} e(\alpha n+f(n))\right| \leq 1+2(q-1) \sum_{\lambda \leq \frac{\log x}{\log q}} q^{\gamma_{q}(f)(\lambda+1)+1} \ll_{q} x^{\gamma_{q}(f)},
$$

as claimed, with $\gamma_{q}(f) \leq 1-\frac{4\|(q-1) f(1)\|^{2}}{q(q+\sqrt{2}-1)^{2} \log q}$ thanks to 16 , Lemme 11].

Case 2. If $f \in \mathcal{F} \backslash \mathcal{F}_{0}$, we follow the same steps as in the previous subcase and we remark, according to 16 , Lemme 8], that $\gamma_{q}(f) \leq 1-\frac{16}{q^{2}(q-1) \log q} \sigma_{q}(f)$ which is clearly satisfactory since $\sigma_{q}(f)=0 \Longleftrightarrow f \in \mathcal{F}_{0}$.

Remark 2.3. We want to note that Theorem 2.2 reformulates 7 , Corollary 1]. Indeed, this corollary states that if $x_{0}, \ldots, x_{q-1}$ are complex numbers sufficiently close to the real axis, with $x_{0}+\cdots+x_{q-1} \neq 1$ then there exists some function $G\left(x_{0}, \ldots, x_{q-1}, t\right)$ that is analytic in $x_{0}, \ldots, x_{q-1}$ and continuous and periodic (of period 1) in $t$ such that

$$
\begin{aligned}
\sum_{0<n<N} x_{0}^{|n|_{0}} x_{1}^{|n|_{1}} \cdots x_{q-1}^{|n|_{q-1}}= & G\left(x_{0}, \ldots, x_{q-1}, \log _{q} N\right) \cdot\left(x_{0}+\cdots+x_{q-1}\right)^{\log _{q} N} \\
& -\frac{x_{1}+\cdots+x_{q-1}}{x_{0}+\cdots+x_{q-1}-1} .
\end{aligned}
$$

This corresponds to the estimate 2.2 for $\alpha=0, x_{i}=e(f(i)), \forall i \in\{0, \ldots, q-1\}$ and $f \in \mathcal{F}_{0}$ with $f(0)=0$ and $f(1) \in \mathbb{Z}$. More precisely,

$$
G\left(x_{0}, \ldots, x_{q-1}, t\right)=x_{0}^{1-\{t\}}\left(1-\frac{q^{1-\{t\}}\left(1-x_{0}\right)}{1-q x_{0}}\right) .
$$

We can check the analyticity of the function $G$, in terms of $x_{0}, \ldots, x_{q-1}$, and its continuity and periodicity, in terms of $t$, by elementary methods. We finally remark that other assumptions on $f$ lead to the estimate (2) of [7, Corollary 1].

Hereafter, we shall resume the study of Gelfond on the class of strongly $q$-additive functions. The result is compared to the main theorem in $[15$, Theorem A] (for $\ell=1$ ) and is the following

Theorem 2.4. Let $q \geq 2, r, b \geq 1, l$ and $a$ be integers and let $f \in \mathcal{F}$ with integer values such that $f(0)=0$. If we denote by

$$
\Xi=|\{1 \leq n \leq x: n \equiv l \bmod r, f(n) \equiv a \bmod b\}| .
$$


Then, we have

$$
\Xi=\frac{x}{r b} \sum_{(j, h) \in J_{1}} \cos \left(2 \pi\left(\frac{l}{r} j+\frac{a}{b} h\right)\right)+O_{q}\left(x^{1-\theta_{f, b, q}}\right)
$$

for some positive constant $\theta_{f, b, q}$, where

$$
J_{1}=\left\{(j, h): 0 \leq j<r, 0 \leq h<b, \frac{h}{b} f \in \mathcal{F}_{0}, \frac{h}{b}(q-1) f(1) \in \mathbb{Z} \text { and }\left(\frac{j}{r}+\frac{h}{b} f(1)\right) \in \mathbb{Z}\right\} .
$$

Proof. We set $H_{0}=\left\{0 \leq h<b, \frac{h}{b} f \in \mathcal{F}_{0}\right\}, H_{1}=\left\{h \in H_{0}, \frac{h}{b}(q-1) f(1) \in \mathbb{Z}\right\}, H_{2}=\{h \in$ $\left.H_{0}, \frac{h}{b}(q-1) f(1) \in \mathbb{R} \backslash \mathbb{Z}\right\}$ and $H_{3}=\left\{0 \leq h<b, \frac{h}{b} f \in \mathcal{F} \backslash \mathcal{F}_{0}\right\}$. Then, we write

$$
\begin{aligned}
\Xi & =\sum_{\substack{n \leq x \\
n \equiv l \bmod r \\
f(n) \equiv a \bmod b}} 1=\frac{1}{r b} \sum_{j=0}^{r-1} e\left(-\frac{l}{r} j\right) \sum_{h=0}^{b-1} e\left(-\frac{a}{b} h\right) \sum_{n \leq x} e\left(\frac{j}{r} n+\frac{h}{b} f(n)\right) \\
& =S_{1}+O\left(S_{2}\right)+O\left(S_{3}\right),
\end{aligned}
$$

where

$$
S_{1}=\frac{1}{r b} \sum_{j=0}^{r-1} e\left(-\frac{l}{r} j\right) \sum_{h \in H_{1}} e\left(-\frac{a}{b} h\right) \sum_{n \leq x} e\left(\frac{j}{r} n+\frac{h}{b} f(n)\right)
$$

and

$$
S_{i}=\frac{1}{b} \sum_{h \in H_{i}}\left|\sum_{n \leq x} e\left(\frac{j}{r} n+\frac{h}{b} f(n)\right)\right| \quad \text { for } i \in\{2,3\} .
$$

Whenever $h \in H_{2}$, Theorem 2.2 gives

$$
S_{2} \ll_{q} x^{1-\gamma_{f, b, q}} \quad \text { with } \quad \gamma_{f, b, q}=\frac{4 \min _{h \in H_{2}}\left\|\frac{h}{b}(q-1) f(1)\right\|}{q(q+\sqrt{2}-1)^{2} \log q}>0 .
$$

Similarly, for $h \in H_{3}$, we get

$$
S_{3} \ll_{q} x^{1-\nu_{f, b, q}} \quad \text { with } \quad \nu_{f, b, q}=\frac{16}{q^{2}(q-1) \log q} \min _{h \in H_{3}} \sigma_{q}\left(\frac{h}{b} f\right)>0 .
$$

Furthermore, we set

$$
J_{1}=\left\{(j, h): 0 \leq j<r, h \in H_{1} \text { and }\left(\frac{j}{r}+\frac{h}{b} f(1)\right) \in \mathbb{Z}\right\}
$$

and

$$
J_{2}=\left\{(j, h): 0 \leq j<r, h \in H_{1} \text { and }\left(\frac{j}{r}+\frac{h}{b} f(1)\right) \notin \mathbb{Z}\right\} .
$$

Subsequently, $S_{1}=T_{1}+T_{2}$ with

$$
T_{1}=\frac{1}{r b} \sum_{(j, h) \in J_{1}} e\left(-\frac{l}{r} j-\frac{a}{b} h\right) \sum_{n \leq x} e\left(\frac{j}{r} n+\frac{h}{b} f(n)\right)=\frac{x}{r b} \sum_{(j, h) \in J_{1}} e\left(-\frac{l}{r} j-\frac{a}{b} h\right)+O(1)
$$


and

$$
T_{2}=\frac{1}{r b} \sum_{(j, h) \in J_{2}} e\left(-\frac{l}{r} j-\frac{a}{b} h\right) \sum_{n \leq x} e\left(\frac{j}{r} n+\frac{h}{b} f(n)\right) \ll 1 .
$$

Finally, we set $\theta_{f, b, q}=\min \left(\gamma_{f, b, q}, \nu_{f, b, q}\right)>0$ to get the desired conclusion.

Remark 2.5. It is worth noticing that, in several cases, the three sets $H_{1}, H_{2}$ and $H_{3}$ could be all nonempty. For instance, this happens if we consider $b=16, q$ is any even integer and $f$ is the strongly $q$-additive function defined by $f(i)=4(i+2)$ for all $i \in\{1, \ldots, q-1\}$. In this case, we get

$$
H_{1}=\{0,4,8,12\}, \quad H_{2}=\{2,6,10,14\} \quad \text { and } \quad H_{3}=\{1,3,5,7,9,11,13,15\} .
$$

Indeed, $\frac{h}{16} f(i)=\frac{h}{4} i+\frac{1}{2} h$ which is an arithmetic progression modulo 1 if and only if $h \in\{0, \ldots, 15\}$ is even.

Furthermore, for every $h \in H_{1}$ we have $\frac{h}{16} f(1) \in \mathbb{Z}$ so that for any integer $r \geq 2$, $\left(\frac{j}{r}+\frac{h}{16} f(1)\right) \in \mathbb{Z}$ if and only if $r \mid j$ and consequently, for $1 \leq j<r$

$$
J_{1}=\{(0,0),(0,4),(0,8),(0,12)\}, \quad J_{2}=\{(j, 0),(j, 4),(j, 8),(j, 12)\} .
$$

\section{Proofs of Theorems 1.3 and 1.5}

Proof of Theorem 1.3. Set $z=\log x$ and let $F(z)$ denote the product of all primes up to $z$, we write

$$
\begin{aligned}
& \mid\{1 \leq n \leq x, f(n) \text { is } k \text {-free }\} \mid \\
= & \sum_{n \leq x} \mu_{k}(f(n))=\sum_{d \geq 1} \mu(d)\left|\left\{n \leq x, d^{k} \mid f(n)\right\}\right| \\
= & \sum_{d \mid F(z)} \mu(d)\left|\left\{n \leq x, d^{k} \mid f(n)\right\}\right|+O\left(\sum_{d \nmid F(z)}\left|\left\{n \leq x, d^{k} \mid f(n)\right\}\right|\right) \\
= & \sum_{d \mid F(z)} \mu(d)\left|\left\{n \leq x, d^{k} \mid f(n)\right\}\right|+O\left(\sum_{p>z}\left|\left\{n \leq x, p^{k} \mid f(n)\right\}\right|\right) .
\end{aligned}
$$

If we apply Theorem 2.4 using the parameters $r=1, b=d^{k}, a=0$, we get

$$
\left|\left\{n \leq x, d^{k} \mid f(n)\right\}\right|=\frac{x}{d^{k}}\left|\mathcal{J}_{d, k}\right|+O_{q}\left(x^{1-\theta_{f, d^{k}, q}}\right),
$$

where

$$
\begin{aligned}
\mathcal{J}_{d, k} & =\left\{0 \leq h<d^{k}, \frac{h}{d^{k}} f \in \mathcal{F}_{0}, \frac{h}{d^{k}} f(1) \in \mathbb{Z}\right\} \\
& =\left\{0 \leq h<d^{k}, \frac{h}{d^{k}} f(j) \in \mathbb{Z}, \forall j \in\{1, \ldots, q-1\}\right\} .
\end{aligned}
$$


Indeed, the last equality follows from the fact that the sequence $\frac{h}{d^{k}} f(1), \ldots \frac{h}{d^{k}} f(q-1)$ is an arithmetic progression modulo 1 and since $\frac{h}{d^{k}} f(1) \in \mathbb{Z}$ then $\frac{h}{d^{k}} f(j) \in \mathbb{Z}, \forall j \in\{1, \ldots, q-1\}$. As we assumed that $f(j)=1$ for some $j \in\{1, \ldots, q-1\}$ then $\mathcal{J}_{d, k}$ reduces to $\{0\}$ for every $d$ and $k$. So, we obtain

$$
\begin{aligned}
\mid\{1 \leq n \leq x, f(n) \text { is } k \text {-free }\} \mid & =\sum_{d \mid F(z)} \mu(d)\left\{\frac{x}{d^{k}}+O\left(x^{1-\theta_{f, d^{k}, q}}\right)\right\}+O\left(\sum_{p>z} \frac{x}{p^{k}}\right) \\
& =x \sum_{d \mid F(z)} \frac{\mu(d)}{d^{k}}+O\left(\sum_{d \mid F(z)} x^{1-\theta_{f, d^{k}, q}}\right)+O\left(\sum_{p>z} \frac{x}{p^{k}}\right) \\
& =x \sum_{d \geq 1} \frac{\mu(d)}{d^{k}}+O\left(x \sum_{d>z} \frac{1}{d^{k}}\right)+O\left(x \sum_{p>z} \frac{1}{p^{k}}\right) \\
& =\frac{x}{\zeta(k)}+O\left(\frac{x}{\log ^{k-1} x}\right) .
\end{aligned}
$$

Hence, the proof is complete.

Remark 3.1. The assumption $f \in \mathcal{F}_{q}^{1}$ might seem artificial and unnecessary but going back to (3.1), we find that this restriction enables a uniform characterization of the set $\mathcal{J}_{d, k}$ so to avoid cumbersome calculations that would make the statement of Theorem 1.3 unappreciated.

Proof of Theorem 1.5. We keep the same notations and steps as in the previous theorem, so that we are left with

$$
\mid\{p \leq x, f(p) \text { is } k \text {-free }\}\left|=\sum_{d \mid F(z)} \mu(d)\right|\left\{p \leq x, d^{k} \mid f(p)\right\} \mid+O\left(\sum_{\substack{p_{1}>z \\ p_{1} \in \mathbb{P}}}\left|\left\{p \leq x, p_{1}^{k} \mid f(p)\right\}\right|\right) .
$$

According to Theorem 1.4 and since $q=2$ (so that the integer $d_{f, d^{k}, 2}$ defined in (1.9) equals 1), we get

$$
\left|\left\{p \leq x, f(p) \equiv 0 \bmod d^{k}\right\}\right|=\frac{1}{d^{k}} \pi(x)+O\left((\log x)^{3} x^{1-\sigma_{f, d^{k}, 2}}\right) .
$$

Hence, we get

$$
\begin{aligned}
\mid\{p \leq x, f(p) \text { is } k \text {-free }\} \mid & =\sum_{d \mid F(z)} \mu(d)\left\{\frac{\pi(x)}{d^{k}}+O\left((\log x)^{3} x^{1-\sigma_{f, d^{k}, 2}}\right)\right\}+O\left(\sum_{\substack{p_{1}>z \\
p_{1} \in \mathbb{P}}} \frac{\pi(x)}{p_{1}^{k}}\right) \\
& =\pi(x) \sum_{d \geq 1} \frac{\mu(d)}{d^{k}}+O\left(\pi(x) \sum_{d>z} \frac{1}{d^{k}}\right)+O\left(\pi(x) \sum_{\substack{p_{1}>z \\
p_{1} \in \mathbb{P}}} \frac{1}{p_{1}^{k}}\right) \\
& =\frac{\pi(x)}{\zeta(k)}+O\left(\frac{x}{\log ^{k} x}\right) .
\end{aligned}
$$

Here, the last equality follows from the prime number theorem. 
Remark 3.2. It is worth noting that this method is unable to give a suitable estimate for any $q>2$. Indeed, if we apply Theorem 1.4 , the error term will be obviously larger than the main term.

However, Theorem 1.5 holds for $q>2$ assuming that $f(q-1)=1$. Indeed, under this assumption and considering (1.9) we get $d_{f, d^{k}, q}=1$ so we can proceed as done above and get the same estimate.

\section{Distribution of $k$-free numbers in congruence classes}

In this section, we look for the distribution of $k$-free numbers verifying a digital constraint. So, if we denote by

$$
D_{b}(x)=\mid\{n \leq x, n \text { is } k \text {-free, } f(n) \equiv a \bmod b\} \mid,
$$

we obtain the following result.

Theorem 4.1. Let $q, k, b \geq 2, l, a$ be integers such that $(b, q-1)=1$ and let $f$ be $a$ strongly q-additive function with integer values. Then, we have

$$
D_{b}(x)=\frac{x}{b \zeta(k)} \sum_{h \in \mathcal{J}} \cos \left(2 \pi \frac{a}{b} h\right)+O_{q}\left(x^{\widetilde{\theta}_{f, b, q}}\right),
$$

where $\widetilde{\theta}_{f, b, q}=\left[1+(k-1)\left(1-\theta_{f, b, q}\right)\right] / k<1$ and

$$
\mathcal{J}=\left\{0 \leq h<b, \frac{h}{b} f(\ell) \in \mathbb{Z}, \forall \ell \in\{1, \ldots, q-1\}\right\} .
$$

Proof. We have

$$
D_{b}(x)=\sum_{1 \leq n \leq x} \nu(n) \mu_{k}(n)
$$

where

$$
\nu(n)= \begin{cases}1 & \text { if } f(n) \equiv a \bmod b \\ 0 & \text { otherwise }\end{cases}
$$

Therefore, if we set $x_{1}=\left\lfloor x^{1 / k}\right\rfloor$ and $x_{2}<x_{1}$ to be chosen later, we get

$$
\begin{aligned}
D_{b}(x) & =\sum_{1 \leq n \leq x} \nu(n) \sum_{d^{k} \mid n} \mu(d)=\sum_{d=1}^{x_{1}} \mu(d) \sum_{i \leq x / d^{k}} \nu\left(d^{k} i\right) \\
& =\sum_{d=1}^{x_{2}} \mu(d) \sum_{i \leq x / d^{k}} \nu\left(d^{k} i\right)+\sum_{d=x_{2}+1}^{x_{1}} \mu(d) \sum_{i \leq x / d^{k}} \nu\left(d^{k} i\right) .
\end{aligned}
$$

Using Theorem 2.4 (with $r=d^{k}$ ), we can estimate the first sum as follows

$$
\sum_{d=1}^{x_{2}} \mu(d) \sum_{i \leq x / d^{k}} \nu\left(d^{k} i\right)=\sum_{d=1}^{x_{2}} \mu(d)\left[\frac{x}{b d^{k}} \sum_{(0, h) \in J_{1}} \cos \left(2 \pi\left(\frac{a}{b} h\right)\right)+O_{q}\left(x^{1-\theta_{f, b, q}}\right)\right] \text {, }
$$


where $J_{1}=\left\{(0, h): 0 \leq h<b, \frac{h}{b} f \in \mathcal{F}_{0}, \frac{h}{b}(q-1) f(1) \in \mathbb{Z}\right.$ and $\left.\frac{h}{b} f(1) \in \mathbb{Z}\right\}$ according to Theorem 2.4.

But, the assumption $\frac{h}{b}(q-1) f(1) \in \mathbb{Z}$ is equivalent to $\frac{h}{b} f(1) \in \mathbb{Z}$ since $(q-1, b)=1$. Henceforth

$$
J_{1}=\left\{(0, h): 0 \leq h<b, \frac{h}{b} f(\ell) \in \mathbb{Z}, \forall \ell \in\{1, \ldots, q-1\}\right\} .
$$

Recalling the set $\mathcal{J}$ defined in 1.10 , we get

$$
\begin{aligned}
\sum_{d=1}^{x_{2}} \mu(d) \sum_{i \leq x / d^{k}} \nu\left(d^{k} i\right)= & \frac{x}{b} \sum_{h \in \mathcal{J}} \cos \left(2 \pi \frac{a}{b} h\right) \sum_{d=1}^{+\infty} \frac{\mu(d)}{d^{k}}-\frac{x}{b} \sum_{h \in \mathcal{J}} \cos \left(2 \pi \frac{a}{b} h\right) \sum_{d=x_{2}+1}^{+\infty} \frac{\mu(d)}{d^{k}} \\
& +O_{q}\left(x_{2} x^{1-\theta_{f, b, q}}\right) \\
= & \frac{x}{b \zeta(k)} \sum_{h \in \mathcal{J}} \cos \left(2 \pi \frac{a}{b} h\right)+O_{q}\left(x_{2} x^{1-\theta_{f, b, q}}\right)+O_{q}\left(x x_{2}^{1-k}\right)
\end{aligned}
$$

where the last $O$-term above is explained as follows: since the series and the integral are convergent, we get for $M \rightarrow+\infty$,

$$
\left|\sum_{d=x_{2}+1}^{M} \frac{\mu(d)}{d^{k}}\right| \leq \sum_{d=x_{2}+1}^{M} \frac{1}{d^{k}} \leq \int_{x_{2}}^{M} \frac{1}{t^{k}} d t=\frac{x_{2}^{1-k}-M^{1-k}}{k-1} .
$$

Again by the same argument, we might bound the second sum in 4.2 :

$$
\left|\sum_{d=x_{2}+1}^{x_{1}} \mu(d) \sum_{i \leq x / d^{k}} \nu\left(d^{k} i\right)\right| \leq \sum_{d=x_{2}+1}^{x_{1}} \frac{x}{d^{k}} \leq x \int_{x_{2}}^{x_{1}} \frac{1}{t^{k}} d t \leq x \frac{x_{2}^{1-k}}{k-1}=O\left(x x_{2}^{1-k}\right) .
$$

Finally, setting $x_{2}=\left\lfloor x^{\theta_{f, b, q} / k}\right\rfloor$, we obtain 4.1 with

$$
\widetilde{\theta}_{f, b, q}=\frac{1+(k-1)\left(1-\theta_{f, b, q}\right)}{k},
$$

and the proof is complete as $\theta_{f, b, q}>0$.

Next, we prove a similar estimate over primes:

Theorem 4.2. Let $q, b \geq 2$ be integers such that $(b, q-1)=1$. Let $f \in \mathcal{F}_{q}^{+}$, then for every $a, c \in \mathbb{Z}$, we have

$$
\mid\{p \leq x, p+c \text { is } k \text {-free, } f(p) \equiv a \bmod b\} \mid=\frac{\beta_{\mathrm{Ar}}}{b} \pi(x)+O\left(\frac{x}{\log ^{2} x}\right),
$$

where $\beta_{\mathrm{Ar}}$ is the Artin's k-dimensional constant defined in (1.3). 
Proof. Let $a, c \in \mathbb{Z}$, we have

$$
\sum_{\substack{p \leq x \\ p+c k \text { free } \\ f(p) \equiv a \bmod b}} 1=\sum_{\substack{p \leq x \\ f(p) \equiv a \bmod b}} \mu_{k}(p+c)=\sum_{\substack{p \leq x \\ f(p) \equiv a \bmod b}} \sum_{d^{k} \mid p+c} \mu(d)=\sum_{d \leq x^{1 / k}} \mu(d) \sum_{\substack{p \leq x \\ p \equiv-c \bmod d^{k} \\ f(p) \equiv a \bmod b}} 1 .
$$

Since $(b, q-1)=1$, we use Theorem 1.6 to obtain

$$
\sum_{\substack{p \leq x \\ p-c \bmod d^{k}}} 1=\frac{1}{b} \pi\left(x ; d^{k},-c\right)+O\left((\log x)^{3} x^{1-\sigma_{f, b, q}}\right) .
$$

Then, we can express 4.3 as follows:

$$
\begin{aligned}
& \sum_{d \leq x^{1 / k}} \mu(d) \sum_{\substack{p \leq x \\
p \equiv-c \bmod d^{k} \\
f(p) \equiv a \bmod b}}=\sum_{d \leq x^{1 / k}} \mu(d)\left(\frac{1}{b} \pi\left(x ; d^{k},-c\right)+O\left((\log x)^{3} x^{\left.1-\sigma_{f, b, q}\right)}\right)\right. \\
&= \sum_{d \leq \log x} \mu(d)\left(\frac{\pi(x)}{b \varphi\left(d^{k}\right)}+O\left(\frac{x}{\log ^{3} x}\right)\right) \\
&+O\left(\sum_{d \leq \log x}(\log x)^{3} x^{1-\sigma f, b, q}\right)+O\left(\sum_{\log x<d \leq x^{1 / k}} \pi\left(x ; d^{k},-c\right)\right) \\
&= \frac{1}{b} \sum_{d \geq 1} \frac{\mu(d)}{\varphi\left(d^{k}\right)} \pi(x)+O\left(\sum_{d>\log x} \frac{\pi(x)}{\varphi\left(d^{k}\right)}+\frac{x}{\log ^{2} x}+\sum_{d>\log x} \frac{x}{d^{k}}\right) \\
&= \frac{1}{b} \prod_{p \in \mathbb{P}}\left(1-\frac{1}{p^{k}(p-1)}\right) \pi(x)+O\left(\frac{x}{\log ^{2} x}\right),
\end{aligned}
$$

where we used the trivial estimate $\pi\left(x ; d^{k},-c\right) \ll x / d^{k}$.

\section{5. $k$-freeness of $f(\mathcal{P}(n))$}

Our goal in this section is to provide an asymptotic formula for

$$
\mid\{n \leq x, f(\mathcal{P}(n)) \text { is } k \text {-free }\} \mid .
$$

We start with two introductory lemmas that will be needed later. A positive integer $n$ is said to be $y$-smooth if $\mathcal{P}(n) \leq y$. Let

$$
\psi(x, y)=\mid\{n \leq x, n \text { is } y \text {-smooth }\} \mid
$$

The following estimate is a simplified version of [26, Theorem 1, Chapter III.5]. 
Lemma 5.1. Let $u=\frac{\log x}{\log y}$, where $x \geq y \geq 2$, then the following bound holds

$$
\psi(x, y) \ll x \exp \left(\frac{-u}{2}\right) .
$$

In what follows, we denote by $\mathbb{P}[w, x]$ the set of primes $p$ such that $w \leq p \leq x$. If the parameters $x \geq y \geq 2$ are fixed throughout the rest of the section, we put

$$
L_{m}=\max \{y, \mathcal{P}(m)\} \text { for all } m \geq 1, \quad \mathbb{P}_{m}=\mathbb{P}\left[L_{m}, x / m\right] .
$$

Lemma 5.2. (see [3, Lemma 3]) Let $x \geq y \geq 2$ and let $h$ and $g$ be two arithmetical functions satisfying $\max \{|h(k)|,|g(k)|\} \leq 1$ for all positive integers $k$, then we have

$$
\sum_{n \leq x} h(\mathcal{P}(n)) g(n)=\sum_{m \leq x / y} \sum_{p \in \mathbb{P}_{m}} h(p) g(m p)+O(\psi(x, y)) .
$$

We start by proving the following

Theorem 5.3. Let $k \geq 2$ be an integer, let $f \in \mathcal{F}_{2}^{+}$(see (1.8). Then, there exists a constant $C_{0}>0$ such that for any $C \leq C_{0}$, the following estimate holds

$$
\mid\{n \leq x, f(\mathcal{P}(n)) \text { is } k \text {-free }\} \mid=\frac{x}{\zeta(k)}+O\left(x \log x \exp \left(-C \log ^{1 / 3} x\right)\right) .
$$

Proof. For every positive integer $e$, we consider the functions $1(e)=1$ and

$$
h(e)= \begin{cases}1 & \text { if } f(e) \text { is } k \text {-free } \\ 0 & \text { else }\end{cases}
$$

Then, for any real parameters $x \geq y \geq 2$ to be chosen later, we have, by Lemma 5.2 ,

$$
\begin{aligned}
\sum_{\substack{n \leq x \\
f(\mathcal{P}(n)) k \text {-free }}} 1 & =\sum_{n \leq x} h(\mathcal{P}(n)) 1(n) \\
& =\sum_{m \leq x / y} \sum_{p \in \mathbb{P}_{m}} h(p) 1(m p)+O(\psi(x, y)) \\
& =\sum_{m \leq x / y} \sum_{\substack{p \in \mathbb{P}_{m} \\
f(p) k \text {-free }}} 1+O(\psi(x, y)) .
\end{aligned}
$$

For any integer $m$ such that $m L_{m} \leq x$, we have

$$
\sum_{\substack{p \in \mathbb{P}_{m} \\ f(p) k \text {-free }}} 1=\sum_{p \leq x / m} \mu_{k}(f(p))-\sum_{p \leq L_{m}} \mu_{k}(f(p))+O(1),
$$

but the sum $\sum_{p \leq x} \mu_{k}(p+c)$ was investigated in Theorem 1.5 giving

$$
\sum_{p \leq x} \mu_{k}(f(p))=\frac{\pi(x)}{\zeta(k)}+O\left(\frac{x}{\log ^{k} x}\right) .
$$


We observe that the error term in $(5.2)$ is an increasing function of $x$. Thus,

$$
\sum_{\substack{p \in \mathbb{P}_{m} \\ f(p) k \text {-free }}} 1=\frac{1}{\zeta(k)}\left(\pi\left(\frac{x}{m}\right)-\pi\left(L_{m}\right)\right)+O\left(\frac{x}{m \log ^{k} \frac{x}{m}}\right) .
$$

From the prime number theorem, we have

$$
\pi(x)=\operatorname{Li}(x)+O(x \exp (-a \sqrt{\log x}))
$$

where $a$ is a positive constant. Hence, (5.3) is rewritten as

$$
\sum_{\substack{p \in \mathbb{P}_{m} \\ f(p) k \text {-free }}} 1=\frac{1}{\zeta(k)}\left(\operatorname{Li}\left(\frac{x}{m}\right)-L i\left(L_{m}\right)\right)+O\left(\frac{x}{m \log ^{k} \frac{x}{m}}\right)+O\left(\frac{x}{m} \exp \left(-a \sqrt{\log \frac{x}{m}}\right)\right) .
$$

Then,

$$
\sum_{\substack{n \leq x \\ f(P(n)) k \text {-free }}} 1=\frac{1}{\zeta(k)} \sum_{m \leq x / y}\left(L i\left(\frac{x}{m}\right)-L i\left(L_{m}\right)\right)+O\left(\psi(x, y)+R_{1}+R_{2}\right),
$$

where

$$
R_{1}=\sum_{m \leq x / y}\left(\frac{x}{m \log ^{k} \frac{x}{m}}\right), \quad R_{2}=\sum_{m \leq x / y} \frac{x}{m} \exp \left(-a \sqrt{\log \frac{x}{m}}\right) .
$$

The same arguments, applied as in (5.1) with $h(e)=1(e)$, lead to the identity

$$
\lfloor x\rfloor=\sum_{n \leq x} 1=\sum_{m \leq x / y}\left(\operatorname{Li}\left(\frac{x}{m}\right)-L i\left(L_{m}\right)\right)+O\left(\psi(x, y)+R_{2}\right) .
$$

Hence,

$$
\sum_{\substack{n \leq x \\ f(\mathcal{P}(n)) k \text {-free }}} 1=\frac{x}{\zeta(k)}+O\left(\psi(x, y)+R_{1}+R_{2}\right) .
$$

Going back to $R_{1}$ and $R_{2}$, we get by elementary estimates

$$
R_{1}=O\left(\frac{x \log x}{\log ^{k} y}\right), \quad R_{2}=O(x \log x \exp (-a \sqrt{\log y})) .
$$

From Lemma 5.1, we have $\psi(x, y)=O\left(x \exp \left(-\frac{\log x}{2 \log y}\right)\right)$. So, by choosing

$$
y=\exp \left(\left(\frac{\log x}{2 a}\right)^{2 / 3}+O\left(\log ^{2 / 3} x \log \log x\right)\right),
$$

we prove that the error term in $(5.4)$ is

$$
O\left(x \log x \exp \left(-C_{0} \log ^{1 / 3} x\right)\right),
$$

where $C_{0}=a / \sqrt{2}$. 
Using the same arguments as in the proof of the previous theorem, we obtain the following result.

Theorem 5.4. Let $q, k, b \geq 2$ be integers such that $(b, q-1)=1$. Let $f \in \mathcal{F}_{q}^{+}$, then for every $a, c \in \mathbb{Z}$, we have

$$
\mid\{n \leq x, \mathcal{P}(n)+c \text { is } k \text {-free, } f(\mathcal{P}(n)) \equiv a \bmod b\} \mid=\frac{\beta_{\mathrm{Ar}}}{b} x+O\left(\frac{x}{\log ^{1 / 3} x}\right),
$$

where $\beta_{\mathrm{Ar}}$ is the Artin's k-dimensional constant defined in (1.3).

Proof. We set

$$
\mathcal{B}=\{n \leq x, \mathcal{P}(n)+c \text { is } k \text {-free, } f(\mathcal{P}(n)) \equiv a \bmod b\}
$$

By applying Lemma 5.2, we get

$$
\begin{aligned}
& |\mathcal{B}|=\sum_{\begin{array}{c}
n \leq x \\
\mathcal{P}(n)+c k \text { free } \\
f(\mathcal{P}(n)) \equiv a \bmod b
\end{array}} 1=\sum_{n \leq x} h(\mathcal{P}(n)) 1(n)
\end{aligned}
$$

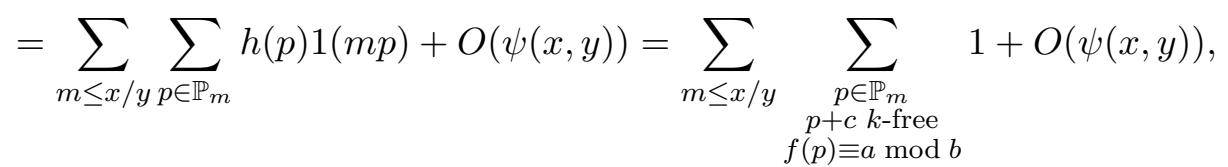

where we set for every $\ell \in \mathbb{N}$,

$$
h(\ell)= \begin{cases}1 & \text { if } \ell+c \text { is } k \text {-free and } f(\ell) \equiv a \bmod b \\ 0 & \text { otherwise }\end{cases}
$$

and $1(\ell)=1$. According to Theorem 4.2 .

$$
\sum_{\substack{p \in \mathbb{P}_{m} \\ p+c k \text { free } \\ f(p) \equiv a \bmod b}} 1=\frac{\beta_{\mathrm{Ar}}}{b}\left(\pi\left(\frac{x}{m}\right)-\pi\left(L_{m}\right)\right)+O\left(\frac{x}{m \log ^{2} \frac{x}{m}}\right),
$$

which could be handled in the same way as in the proof of Theorem 5.3 to get the desired conclusion.

\section{Acknowledgments}

The first author would like to thank the Analytic Number Theory team in Institut Élie Cartan de Lorraine (Nancy) for the nice atmosphere of work that was provided during his stay in Nancy. The authors are also very grateful to the referee for a careful reading of the manuscript that contributed to the improvement of this work. 


\section{References}

[1] R. C. Baker and J. Pintz, The distribution of squarefree numbers, Acta Arith. 46 (1985), no. $1,73-79$.

[2] R. C. Baker and K. Powell, The distribution of $k$-free numbers, Acta Math. Hungar. 126 (2010), no. 1-2, 181-197.

[3] W. D. Banks, G. Harman and I. E. Shparlinski, Distributional properties of the largest prime factor, Michigan Math. J. 53 (2005), no. 3, 665-681.

[4] R. Bellman and H. N. Shapiro, On a problem in additive number theory, Ann. of Math. (2) 49 (1948), 333-340.

[5] J. Coquet, Sur les fonctions $Q$-multiplicatives et $Q$-additives, Thèse $3^{\text {ème }}$ cycle, Orsay, 1975.

[6] C. J. de la Vallée Poussin, Recherches analytiques sur la théorie des nombres premiers, Brux. S. sc. 21, 1896.

[7] M. Drmota and C. Mauduit, Weyl sums over integers with affine digit restrictions, J. Number Theory 130 (2010), no. 11, 2404-2427.

[8] A. O. Gel'fond, Sur les nombres qui ont des propriétés additives et multiplicatives données, Acta Arith. 13 (1968), 259-265.

[9] S. W. Graham, The distribution of squarefree numbers, J. London Math. Soc. (2) 24 (1981), no. 1, 54-64.

[10] S. W. Graham and J. Pintz, The distribution of $r$-free numbers, Acta Math. Hungar. 53 (1989), no. 1-2, 213-236.

[11] J. Hadamard, Sur la distribution des zéros de la fonction $\zeta(s)$ et ses conséquences arithmétiques, Bull. Soc. Math. France 24 (1896), 199-220.

[12] D. R. Heath-Brown, Power-free values of polynomials, Q. J. Math. 64 (2013), no. 1, 177-188.

[13] C. H. Jia, The distribution of squarefree numbers, Beijing Daxue Xuebao (1987), no. 3, 21-27.

[14] _ The distribution of square-free numbers, Sci. China Ser. A 36 (1993), no. 2, 154-169. 
[15] D.-H. Kim, On the joint distribution of q-additive functions in residue classes, J. Number Theory 74 (1999), no. 2, 307-336.

[16] B. Martin, C. Mauduit and J. Rivat, Théorème des nombres premiers pour les fonctions digitales, Acta Arith. 165 (2014), no. 1, 11-45.

[17] , Fonctions digitales le long des nombres premiers, Acta Arith. 170 (2015), no. $2,175-197$.

[18] C. Mauduit, A. Sárközy, On the arithmetic structure of sets characterized by sum of digits properties, J. Number Theory 61 (1996), no. 1, 25-38.

[19] X. Meng, The distribution of $k$-free numbers and the derivative of the Riemann zetafunction, Math. Proc. Cambridge Philos. Soc. 162 (2017), no. 2, 293-317.

[20] L. Mirsky, The number of representations of an integer as the sum of a prime and a $k$-free integer, Amer. Math. Monthly 56 (1949), 17-19.

[21] M. Mkaouar, N. Ouled Azaiez and J. M. Thuswaldner, Sur les chiffres des nombres premiers translatés, Funct. Approx. Comment. Math. 51 (2014), no. 2, 237-267.

[22] H. L. Montgomery and R. C. Vaughan, The distribution of squarefree numbers, in: Recent Progress in Analytic Number Theory, Vol. 1, (Durham, 1979), 247-256, Academic Press, London, 1981.

[23] F. Pappalardi, A survey on k-freeness, in: Number Theory, 71-88, Ramanujan Math. Soc. Lect. Notes Ser. 1, Ramanujan Math. Soc., Mysore, 2005.

[24] T. Reuss, Power-free values of polynomials, Bull. Lond. Math. Soc. 47 (2015), no. 2, $270-284$.

[25] J. Sándor, D. S. Mitrinović and B. Crstici, Handbook of Number Theory I, Springer, Dordrecht, 2006.

[26] G. Tenenbaum, Introduction to Analytic and Probabilistic Number Theory, Cambridge Studies in Advanced Mathematics 46, Cambridge University Press, Cambridge, 1995.

[27] I. M. Vinogradov, The method of trigonometrical sums in the theory of numbers, Dover Publications, Mineola, NY, 2004.

[28] A. Walfisz, Weylsche Exponentialsummen in der neueren Zahlentheorie, Mathematische Forschungsberichte, XV, VEB Deutscher Verlag der Wissenschaften, Berlin, 1963. 
Karam Aloui

Université de Lorraine, Institut Élie Cartan de Lorraine, UMR 7502, Campus Aiguillettes, BP 70739, 54506 Vandœvre-lès-Nancy cedex, France and

Université de Sfax, Faculté des Sciences de Sfax, Route Soukra, B.P. 1171, Sfax 3000, Tunisie

E-mail address: karam.aloui@univ-lorraine.fr

Mohamed Mkaouar

Université de Sfax, Faculté des Sciences de Sfax, Route Soukra, B.P. 1171, Sfax 3000, Tunisie

E-mail address: mohamed.mkaouar@fss.rnu.tn

Walid Wannes

Institute of Analysis and Number Theory, Graz University of Technology, A-8010 Graz, Steyrergasse 30/II, Austria and Université de Sfax, Faculté des Sciences de Sfax, Route Soukra, B.P. 1171, Sfax 3000, Tunisie

E-mail address: wannes.walid.fss@gmail.com, wannes@math.tugraz.at 